Publ. RIMS, Kyoto Univ.

19 (1983), 305-316

\title{
A Cut-Free Sequential System for the Propositional Modal Logic of Finite Chains
}

By

\author{
Hirokazu NisHimuRA*
}

\begin{abstract}
The main purpose of this paper is to give a cut-free Gentzen-type sequential system for $\mathbf{K} 4.3 \mathrm{G}$ of finite chains. The cut-elimination theorem is proved both modeltheoretically and proof-theoretically.
\end{abstract}

\section{$\S 1$. Introduction}

There are thousands of modal logics, only a bit of which enjoy Gentzen-type sequential formulations. Modal logics with cut-free sequential systems are even fewer and it is often a challenging problem to find out such pleasant formulations to a given modal logic. See Zeman [7] for the general reference and Sato [5] for an example of recent such attempts. The main purpose of this paper is to give a cut-free sequential system for $\mathbb{K} 4.3 \mathbf{G}$ of Gabbay [2, §25].

Formulas (of $\mathbb{K} 4.3 \mathbf{G}$ ) are constructed from propositional variables $p$ and $\perp$ (falsity) by using $\supset$ (implication) and $\square$ (necessity). Other connectives like $\wedge$ (conjunction), $\vee$ (disjunction) and $\neg$ (negation) can be introduced as defined symbols in the usual manner. A structure (for $\mathbb{K} 4.3 G)$ is a quadruple $\left(S, R, s_{0}, D_{s}\right)$, where

(1) $S$ is a nonempty finite set.

(2) $R$ is an irreflexive transitive binary relation on $S$ such that either $x R y$ or $y R x$ for any distinct $x, y \in S$.

(3) $s_{0} \in S$.

(4) For any $s \in S, D_{s}$ assigns a truth-value 0 or 1 to every propositional variable.

Given a structure $\left(S, R, s_{0}, D_{s}\right)$, the truth-value $\|A\|_{s}$ of a formula $A$ at $s \in S$ is defined inductively as follows:

Received May 24, 1982.

* Research Institute for Mathematical Sciences, Kyoto University, Kyoto 606, Japan. 
(1) $\|p\|_{s}=D_{s}(p)$ for any propositional variable $p$.

(2) $\|\perp\|_{s}=0$.

(3) $\|A \supset B\|_{s}=1$ iff $\|A\|_{s}=0$ or $\|B\|_{s}=1$.

(4) $\|\square A\|_{s}=1$ iff for any $t \in S$ such that $s R t,\|A\|_{t}=1$. $\vDash A$.

If $\|A\|_{s_{0}}=1$ for any structure $\left(S, R, s_{0}, D_{s}\right)$, then $A$ is called valid, notation:

K4.3G can be axiomatized by the classical propositional calculus plus the following axioms and inference rules.

(A1) $\square(A \supset B) \supset(\square A \supset \square B)$

(A2) $\square A \supset \square \square A$

(A3) $\quad \square(\square A \supset A) \supset \square A$

(A4) $\quad \square(\square A \supset B) \vee \square(B \wedge \square B \supset A)$

(R1) $\frac{A}{\square A}$

We write $\vdash_{\mathrm{K} 4.3 \mathrm{G} A} A$ if $A$ is provable in the above formal system.

Theorem 1.1. For any formula $A, \vdash$ K4.3G $A$ iff $\vDash A$.

In the next section we present our sequential system SK4.3G and establish its cut-freeness semantically while its purely syntactic proof is given in Section 3. Finally we admit that this paper was inspired by a cut-free sequential system of Leivant [4] for the modal logic $\mathbb{K} 4 \mathbf{G}$ of finite partial orders but its subtle error in the proof of the cut-elimination theorem is corrected in our more general context.

\section{§2. Cut-free System for $\mathrm{K} 4.3 \mathrm{G}$}

A sequent is an ordered pair $(\Gamma, \Delta)$ of (possibly empty) finite sets of formulas, which we usually denote by $\Gamma \rightarrow \Delta$. We use such self-explanatory notations as $A, \Gamma \rightarrow \Delta, B$ for $\{A\} \cup \Gamma \rightarrow \Delta \cup\{B\}$ and $\square \Gamma$ for $\{\square A: A \in \Gamma\}$ freely.

Our sequential formal system SK4.3G ("S" for "Sequential") consists of the following axioms and inference rules:

Axioms : $A \rightarrow A$

$\perp \rightarrow$

Rules: $\frac{\Gamma \rightarrow \Delta}{\Pi, \Gamma \rightarrow \Delta, \Lambda}$ (thin)

$$
\begin{aligned}
& \frac{\Gamma \rightarrow \Delta, A \quad B, \Pi \rightarrow \Lambda}{A \supset B, \Gamma, \Pi \rightarrow \Delta, \Lambda} \quad(\supset \mathrm{L}) \\
& \frac{A, \Gamma \rightarrow \Delta, B}{\Gamma \rightarrow \Delta, A \supset B} \quad(\supset \mathrm{R})
\end{aligned}
$$




$$
\frac{\{\Gamma, \square \Gamma, \square \Pi \rightarrow \Pi, \square \Lambda: \Pi \cup \Lambda=\Delta, \Pi \cap \Lambda=\varnothing \text { and } \Pi \neq \varnothing\}}{\square \Gamma \rightarrow \square \Delta}
$$

, where $\Delta \neq 0$ in (GL4.3).

It is easy to show that the following rules are admissible in $\mathbf{S K 4 . 3 G}$.

$$
\begin{aligned}
& \frac{\Gamma \rightarrow \Delta, A}{\neg A, \Gamma \rightarrow \Delta}(\neg \mathrm{L}) \\
& \frac{A, \Gamma \rightarrow \Delta}{\Gamma \rightarrow \Delta, \neg A} \quad(\neg \mathrm{R}) \\
& \left.\begin{array}{r}
-\frac{A, \Gamma \rightarrow \Delta}{A \wedge B, \Gamma \rightarrow \Delta} \\
\frac{B, \Gamma \rightarrow \Delta}{A \wedge B, \Gamma \rightarrow \Delta}
\end{array}\right\} \quad(\wedge \mathrm{L}) \\
& \frac{\Gamma \rightarrow \Delta, A \quad \Gamma \rightarrow \Delta, B}{\Gamma \rightarrow \Delta, A \wedge B} \quad(\wedge \mathrm{R}) \\
& \frac{A, \Gamma \rightarrow \Delta \quad B, \Gamma \rightarrow \Delta}{A \vee B, \Gamma \rightarrow \Delta} \\
& \left.\begin{array}{l}
\Gamma \rightarrow \Delta, A \\
\qquad \rightarrow \Delta, A \vee B \\
\begin{array}{l}
\Gamma \rightarrow \Delta, B \\
\Gamma \rightarrow \Delta, A \vee B
\end{array}
\end{array}\right\}
\end{aligned}
$$

If $\Gamma \rightarrow \Delta$ is provable in $\operatorname{SK} 4.3 \mathrm{G}$, we write $\vdash \operatorname{sk} 4.3 \mathrm{G} \Gamma \rightarrow \Delta$. We notice that the rule (GL4.3) has the variable number of upper sequents, depending on the number $|\Delta|$. If $|\Delta|=1$, our rule (GL4.3) degenerates into the rule (GL) of Leivant [4].

$$
\frac{\Gamma, \square \Gamma, \square A \rightarrow A}{\square \Gamma \rightarrow \square A}
$$

If $|\Delta|=2$, then the rule (GL4.3) goes as follows:

$$
\frac{\Gamma, \square \Gamma, \square A, \square B \rightarrow A, B \quad \Gamma, \square \Gamma, \square A \rightarrow A, \square B \quad \Gamma, \square \Gamma, \square B \rightarrow \square A, B}{\square \Gamma \rightarrow \square A, \square B}
$$

To deepen the reader's understanding of the rule (GL4.3), we shall show that $\vdash \mathbf{S K 4 . 3 G} \rightarrow \square(\square A \supset B) \vee \square(B \wedge \square B \supset A)$.

We have the following proof $\pi_{1}$ of the sequent $\square(\square A \supset B), \square(B \wedge \square B \supset A)$ $\rightarrow \square A \supset B, B \wedge \square B \supset A$.

$$
\begin{aligned}
& \frac{B \rightarrow B}{\square(\square A \supset B), \square(B \wedge \square B \supset A), \square A, B \rightarrow A, B} \quad \text { (thin) } \\
& \bar{\square}(\square \bar{A} \supset B), \square(B \wedge \square B \supset A), \square A, B \wedge \square B \rightarrow A, B \quad(\wedge \mathrm{L}) \\
& \frac{\square(\square A \supset B), \square(B \wedge \square B \supset A), \square A \rightarrow B, B \wedge \square B \supset A}{\square(\square A \supset B), \square(B \wedge \square B \supset A) \rightarrow \square A \supset B, B \wedge \square B \supset A} \quad(\supset \mathrm{R})
\end{aligned}
$$


We have the following proof $\pi_{2}$ of the sequent $\square(\square A \supset B)$ $\rightarrow \square A \supset B, \square(B \wedge \square B \supset A)$.

$$
\begin{aligned}
& \begin{array}{r}
A \rightarrow A \\
\square(\square A \supset B), \square A \supset B, \square A, A, \square(B \wedge \square B \supset A), B \wedge \sqsupset B \rightarrow A
\end{array} \text { (thin) }
\end{aligned}
$$

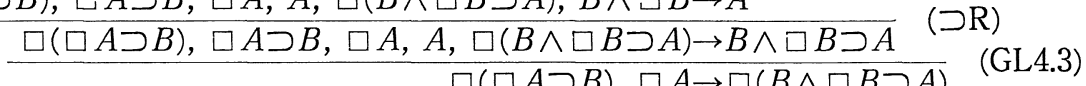

$$
\begin{aligned}
& \begin{array}{ll}
\frac{\square(\square A \supset B), \square A \rightarrow \square(B \wedge \square B \supset A)}{\square(\square A \supset B), \square A \rightarrow B, \square(B \wedge \square B \supset A)} & \text { (thin) } \\
\frac{\square(\square A \supset B) \rightarrow \square A \supset B, \square(B \wedge \square B \supset A)}{\square(\square)} &
\end{array}
\end{aligned}
$$

We have the following proof $\pi_{3}$ of the sequent $\square(B \wedge \square B \supset A) \rightarrow$ $\square(\square A \supset B), B \wedge \square B \supset A$.

$$
\begin{aligned}
& \begin{array}{r}
B \rightarrow B \\
\square(B \wedge \square B \supset A), B \wedge \square B \supset A, \square B, B, \square(\square A \supset B), \square A \rightarrow B
\end{array} \text { (thin) } \\
& \square(B \wedge \square B \supset A), B \wedge \square B \supset A, \square B, B, \square(\square A \supset B) \rightarrow \square A \supset B \quad(\supset \mathrm{R}) \\
& \square(B \wedge \square B \supset A), \square B \rightarrow \square(\square A \supset B) \\
& \square(B \wedge \square B \supset A), \square B \rightarrow \square(\square A \supset B), A \\
& \square(B \wedge \square B \supset A), B \wedge \square B \rightarrow \square(\square A \supset B), A \\
& \square(B \wedge \square B \supset A) \rightarrow \square(\square A \supset B), B \wedge \square B \supset A \quad(\supset \mathrm{R})
\end{aligned}
$$

Therefore

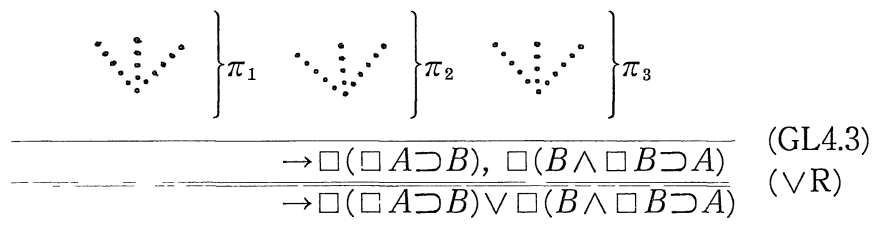

A sequent $\Gamma \rightarrow \Delta$ is called realizable if for some structure $\left(S, R, s_{0}, D_{s}\right)$, $\|A\|_{s_{0}}=1$ for any $A \in \Gamma$ and $\|B\|_{s_{0}}=0$ for any $B \in \Delta$. A sequent $\Gamma \rightarrow \Delta$ which is not realizable is called valid, notation: $\vDash \Gamma \rightarrow \Delta$.

Theorem 2.1. (Soundness Theorem). For any sequent $\Gamma \rightarrow \Delta$, if $\vdash_{\mathrm{sK} 4.3 \mathrm{G}} \Gamma \rightarrow \Delta$, then $\vDash \Gamma \rightarrow \Delta$.

Proof. By induction on a proof of $\Gamma \rightarrow \Delta$.

Corollary 2.2. (Consistency). The empty sequent $\rightarrow$ is not provable in $\mathbf{S K} 4.3 \mathrm{G}$.

Theorem 2.3. (Completeness Theorem). For any sequent $\Gamma \rightarrow \Delta$, if $\vDash \Gamma \rightarrow \Delta$, then $\vdash$ sK4.3G $\Gamma \rightarrow \Delta$.

Proof. Let $\Gamma \rightarrow \Delta$ be the given sequent. We denote by $\Omega$ the set of all subformulas occurring in a formula of $\Gamma \cup \Delta$. A sequent $\Pi \rightarrow \Lambda$ is called $\Omega$ saturated if it satisfies the following conditions:

(1) $\nvdash$ SK4.3G $\Pi \rightarrow \Lambda$.

(2) $\Pi \cup \Lambda \subseteq \Omega$. 
(3) For any $A \in \Omega-(\Pi \cup \Lambda), \vdash$ sk4.3G $\Pi \rightarrow \Lambda, A$ and $\vdash$ SK4.3G $A, \Pi \rightarrow \Lambda$.

Assuming that $\nvdash_{\mathrm{SK} 4.3 \mathrm{G}} \Gamma \rightarrow \Delta$, we shall show that $\not \models \rightarrow \Delta$. We denote by $W(\Omega)$ the set of all $\Omega$-saturated sequents. Since $\vdash$ sK4.3G $\Gamma \rightarrow \Delta$, the sequent $\Gamma \rightarrow \Delta$ can be extended to some $\Gamma_{0} \rightarrow \Delta_{0} \in W(\Omega)$. For any set $\Sigma$ of formulas, $(\Sigma)_{\square}$ denotes the set of all formulas $A$ such that $\square A \in \Sigma$. If $\left(\Delta_{0}\right)_{\square}=\varnothing$, then let $S=\left\{\Gamma_{0} \rightarrow \Delta_{0}\right\}$. If $\left(\Delta_{0}\right) \neq \varnothing, \nvdash$ SK4.3G $\square\left(\Gamma_{0}\right)_{\square} \rightarrow \square\left(\Delta_{0}\right)_{\square}$. Therefore, taking the rule (GL4.3) into consideration, there exist two sets $\Sigma_{1}, \Sigma_{2}$ of formulas such that:

(1) $\Sigma_{1} \neq \varnothing$.

(2) $\Sigma_{1} \cup \Sigma_{2}=\left(\Delta_{0}\right)_{\square}$

(3) $\Sigma_{1} \cap \Sigma_{2}=\emptyset$.

(4) $\nvdash \operatorname{SK4.3G}\left(\Gamma_{0}\right)_{\square}, \square\left(\Gamma_{0}\right)_{\square}, \square \Sigma_{1} \rightarrow \Sigma_{1}, \square \Sigma_{2}$.

The sequent $\left(\Gamma_{0}\right)_{\square}, \square\left(\Gamma_{0}\right)_{\square}, \square \Sigma_{1} \rightarrow \Sigma_{1}, \square \Sigma_{2}$ can be extended to some $\Gamma_{1} \rightarrow \Delta_{1} \in W(\Omega)$. We notice that:

(1) $\left(\Gamma_{0}\right)_{\square} \subset\left(\Gamma_{1}\right)_{\square}$ (By $\subset$ we denote the proper inclusion).

(3) $\left(\Gamma_{0}\right)_{\square} \subseteq \Gamma_{1}$.

(3) $\left(\Delta_{0}\right)_{\square} \subseteq \Delta_{1} \cup\left(\Delta_{1}\right)_{\sqsubset}$.

If $\left(\Delta_{1}\right)_{\square}=\varnothing$, then we let $S=\left\{\Gamma_{0} \rightarrow \Delta_{0}, \Gamma_{1} \rightarrow \Delta_{1}\right\}$. If $\left(\Delta_{1}\right)_{\square} \neq \varnothing$, we repeat the above process. In any case we finally obtain a sequence $\left\{\Gamma_{i} \rightarrow \Delta_{\imath}\right\}_{\imath=0}^{k}$ of $W(\Omega)$ such that:

(1) $\left(\Gamma_{0}\right)_{\square} \subset\left(\Gamma_{1}\right)_{\square} \subset \cdots \subset\left(\Gamma_{k}\right)_{\square}$.

(2) $\left(\Gamma_{i}\right)_{\square} \subseteq \Gamma_{i+1}$ for any $0 \leqq i<k$.

(3) $\left(\Delta_{i}\right)_{\square} \subseteq \Delta_{i+1} \cup\left(\Delta_{i+1}\right)_{\square}$ for any $0 \leqq i \leqq k-1$.

(4) $\left(\Delta_{i}\right)_{\square} \neq \varnothing$ for any $i<k$ and $\left(\Delta_{k}\right)_{\square}=\varnothing$.

Set $S=\left\{\Gamma_{0} \rightarrow \Delta_{0}, \cdots, \Gamma_{k} \rightarrow \Delta_{k}\right\}$. We let $\left(\Gamma_{i} \rightarrow \Delta_{i}\right) R\left(\Gamma_{j} \rightarrow \Delta_{\jmath}\right)$ iff $i<j$. Let $s_{0}=\Gamma_{0} \rightarrow \Delta_{0}$. We define $D_{\Gamma_{i} \rightarrow \Delta_{i}}$ as follows :

$D_{\Gamma_{i} \rightarrow \Delta_{i}}(p)=1$ iff $p \in \Gamma_{i}$ for any propositional variable $p$.

It is not difficult to show by induction on $A \in \Omega$ that for any $0 \leqq i \leqq k$,

(1) $\|A\|_{\Gamma_{i \rightarrow \Delta_{i}}=1}$ if $A \in \Gamma_{i}$.

(2) $\|A\|_{\Gamma_{i} \rightarrow \Delta_{i}}=0 \quad$ if $A \in \Delta_{i}$.

In particular, we can conclude that $\Gamma_{0} \rightarrow \Delta_{0}$ is realizable and so is $\Gamma \rightarrow \Delta$. This completes the proof.

Corollary 2.4. For any formula $A, \vdash_{\mathbf{K} 4.3 \mathrm{G}} A$ iff $\vdash_{\mathrm{SK} 4.3 \mathrm{G}} \rightarrow A$.

Corollary 2.5. The following inference rule is admissible in SK4.3G.

$$
\frac{\Gamma \rightarrow \Delta, A \quad A, \Pi \rightarrow \Lambda}{\Gamma, \Pi \rightarrow \Delta, \Lambda} \text { (cut) }
$$


In the next section we will give a purely syntactical proof of Corollary 2.5.

\section{§3. Cut-Elimination Theorem}

The main purpose of this section is to give a proof-theoretical proof of Corollary 2.5 by amending Gentzen's original proof (for LK and LJ) such as seen in Takeuti [6].

Theorem 3.1. (Cut-elimination Theorem). The following inference is admissible in SK4.3G.

$$
\begin{aligned}
\frac{\Gamma_{1} \rightarrow \Delta_{1}, A \quad A, \Gamma_{2} \rightarrow \Delta_{2}}{\Gamma \rightarrow \Delta} & \text { (cut) } \\
& \left(\Gamma=\Gamma_{1} \cup \Gamma_{2} \text { and } \Delta=\Delta_{1} \cup \Delta_{2}\right)
\end{aligned}
$$

For technical reasons we deal with a slightly modified version of SK4.3G, say SK4.3G', which is obtainable from SK4.3G by restricting rules (thin) and $(\supset \mathrm{L})$ to the following (thin $)_{\square}$ and $(\supset \mathrm{L})^{\prime}$

$$
\begin{aligned}
& \frac{\square(\Gamma)_{\square \rightarrow \square(\Delta)_{\square}}}{\Gamma \rightarrow \Delta} \quad(\text { thin })_{\square} \\
& \frac{\Gamma \rightarrow \Delta, A \quad B, \Gamma \rightarrow \Delta}{A \supset B, \Gamma \rightarrow \Delta} \quad(\supset \mathrm{L})^{\prime}
\end{aligned}
$$

and instead adopting as axioms sequents $\Gamma \rightarrow \Delta$ satisfying at least one of the following conditions :

(1) $p \in \Gamma \cap \Delta$ for some propositional variable $p$.

(2) $\perp \in \Gamma$.

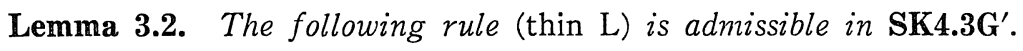

$$
\frac{\Gamma \rightarrow \Delta}{A, \Gamma \rightarrow \Delta} \quad(\text { thin L) }
$$

Proof. It is sufficient to deal with proof figures which contain only one (thin L) as the last inference.

$$
\begin{aligned}
& \because \because \bullet^{\circ} \\
& \frac{\Gamma \rightarrow \Delta}{A, \Gamma \rightarrow \Delta} \text { (thin L) }
\end{aligned}
$$

The proof is carried out by double induction mainly on the formula $A$ and secondly on the length of longest threads of the proof of $\Gamma \rightarrow \Delta$. Here we deal only with a special case of (GL4.3) being the last inference of the proof of $\Gamma \rightarrow \Delta$. 


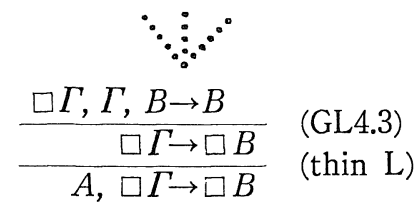

If $A$ is not of the form $\square C$, then (thin $\mathrm{L}$ ) degenerates into (thin) $\square$ which is of course admissible. If $A$ is of the form $\square C$, the above proof figure is transformed into:

$$
\begin{aligned}
& \because \vdots 0^{\circ} \\
& \begin{aligned}
\frac{\square \Gamma, \Gamma, B \rightarrow B}{\square C, \square \Gamma, \Gamma, \square B \rightarrow B} & \text { (thin L) } \\
\frac{\square C, C, \square \Gamma, \Gamma, \square B \rightarrow B}{\square C, \square \Gamma \rightarrow \square B} & \text { (thin L) }
\end{aligned}
\end{aligned}
$$

Therefore the induction process works well.

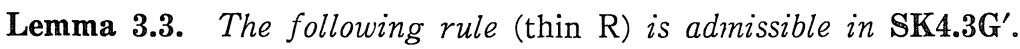

$$
\frac{\Gamma \rightarrow \Delta}{\Gamma \rightarrow \Delta, A} \text { (thin R) }
$$

Proof. It is sufficient to deal with proof figures which contain only one (thin $\mathrm{R}$ ) as the last inference.

$$
\begin{aligned}
& \because \because \vdots 0^{\circ} \\
& \frac{\Gamma \rightarrow \Delta}{\Gamma \rightarrow \Delta, A} \text { (thin R) }
\end{aligned}
$$

The proof is carried out by double induction mainly on the formula $A$ and secondly on the length of longest threads of the proof of $\Gamma \rightarrow \Delta$. Here we deal only with a special case of (GL4.3) being the last inference of the proof of $\Gamma \rightarrow \Delta$.

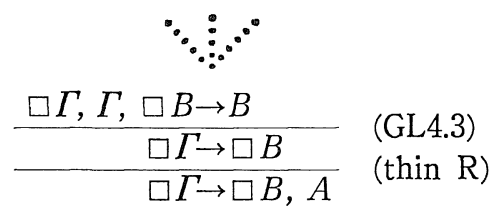

If $A$ is not of the form $\square C$, then (thin $\mathrm{R}$ ) degenerates into (thin) $\square$, which is of course admissible. If $A$ is of the form $\square C$, the above proof figure is trans- 
formed into:

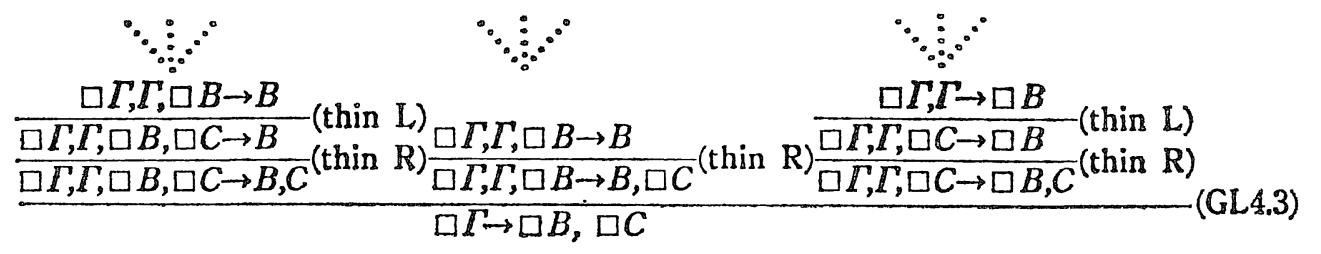

Therefore the induction process works well.

Lemma 3.4. The rule (thin) is admissible in $\mathbf{S K} 4.3 \mathbf{G}^{\prime}$.

Proof. Follows readily from Lemmas 3.2 and 3.3.

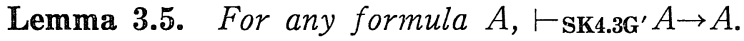

Proof. By induction on $A$. Here we deal only with the case of $A$ being of the form $\square B$.

$$
\begin{array}{cl}
\because \because \vdots 0^{\circ} & \\
\frac{B \rightarrow B}{\square B, B, \square B \rightarrow B} & \text { (thin) } \\
\frac{\square B \rightarrow \square B}{\square B} & \text { (GL4.3) }
\end{array}
$$

Proposition 3.6. For any sequent $\Gamma \rightarrow \Delta, \vdash \mathrm{sK}_{4.3 \mathrm{G}} \Gamma \rightarrow \Delta$ iff $\vdash \mathrm{SK}^{\mathrm{SG}} \mathrm{G}^{\prime} \Gamma \rightarrow \Delta$.

Proof. (1) If part: Trivial. (2) If only part: Use Lemmas 3.4 and 3.5.

Theorem 37. The following inference is admissible in $\mathbf{S K} \mathbf{4} . \mathbf{3} \mathbf{G}^{\prime}$.

$$
\frac{\Gamma_{1} \rightarrow \Delta_{1}, A \quad A, \Gamma_{2} \rightarrow \Delta_{2}}{\Gamma \rightarrow \Delta} \text { (cut) }
$$

$$
\left(\Gamma=\Gamma_{1} \cup \Gamma_{2} \text { and } \Delta=\Delta_{1} \cup \Delta_{2}\right)
$$

Proof. It is sufficient to deal with proof figures which contain only one (cut) as the last inference. Thus we must consider the following proof figure $\pi$.

$$
\begin{aligned}
\frac{\Gamma_{1} \rightarrow \Delta_{1}, A \quad A, \Gamma_{2} \rightarrow \Delta_{2}}{\Gamma \rightarrow \Delta} & \text { (cut) } \\
& \left(\Gamma=\Gamma_{1} \cup \Gamma_{2} \text { and } \Delta=\Delta_{1} \cup \Delta_{2}\right)
\end{aligned}
$$

By the grade of a formula $B$, we mean the number of logical symbols contained in $B$. By $\gamma(\pi)$ we denote the grade of the cut formula $A$. By $\delta_{l}^{1}(\pi)$ we denote the number of formulas of the form $\square B$ that occur as subformulas of formulas in $\Gamma_{1} \cup \Delta_{1}{ }^{1)}$. We denote by $\delta_{l}^{2}(\pi)$ the number of formulas of the form $\square B$ that occur in $\Gamma_{1}{ }^{2}$. Obviously $\delta_{l}^{1}(\pi) \geqq \delta_{l}^{2}(\pi)$. We denote by $\delta_{l}(\pi)$ the number $\delta_{l}^{1}(\pi)-\delta_{l}^{2}(\pi)$. By $\delta_{r}^{1}(\pi)$ we denote the number of formulas of the 
form $\square B$ that occur as subformulas in $\Gamma_{2} \cup \Delta_{2}{ }^{3)}$. We denote by $\delta_{r}^{2}(\pi)$ the number of formulas of the form $\square B$ that occur in $\Gamma_{2}^{4}$. We denote by $\delta_{r}(\pi)$ the number $\delta_{r}^{1}(\pi)-\delta_{r}^{2}(\pi)$. We decree that $\delta(\pi)=\delta_{l}(\pi)+\delta_{r}(\pi)$. By $\rho_{l}(\pi)$ we denote the number of the longest threads that end with the left upper sequent $\Gamma_{1} \rightarrow \Delta_{1}, A$ and contain the cut formula $A$ consecutively. Similarly we denote by $\rho_{r}(\pi)$ the number of the longest threads that end with the right upper sequent $A, \Gamma_{2} \rightarrow \Delta_{2}$ and contain the cut formula $A$ consecutively. By $\rho(\pi)$ we mean the number $\rho_{l}(\pi)+\rho_{r}(\pi)$. Now our proof proceeds by triple induction mainly on $\gamma(\pi)$, secondly on $\delta(\pi)$ and thirdly on $\rho(\pi)$. Since our proof is not by the usual double induction on $\gamma(\pi)$ and $\rho(\pi)$, we should be careful enough even in dealing with classical cases.

(1) $\rho(\pi)=2$ : Since in rule (GL4.3) the antecedent of the lower sequent is contained in that of every upper sequent, $A$ can be of the form $\square B$ only when the right upper sequent $\square B, \Gamma_{2} \rightarrow \Delta_{2}$ is an axiom sequent. In this case $\Gamma \rightarrow \Delta$ is also an axiom sequent. Therefore the only nontrivial case we must consider goes as follows:

$$
\begin{aligned}
& \because \because \because 0^{\circ} \quad \because \because \therefore 0^{\circ} \quad \because \vdots 0^{\circ} \\
& \frac{\frac{A, \Gamma_{1} \rightarrow \Delta_{1}, B}{\Gamma_{1} \rightarrow \Delta_{1}, A \supset B} \quad(\supset \mathrm{R})}{\frac{\Gamma_{2} \rightarrow \Delta_{2}, A \quad B, \Gamma_{2} \rightarrow \Delta_{2}}{A \supset B, \Gamma_{2} \rightarrow \Delta_{2}}} \quad(\supset \mathrm{L})^{\prime} \\
& \left(\Gamma=\Gamma_{1} \cup \Gamma_{2} \text { and } \Delta=\Delta_{1} \cup \Delta_{2}\right)
\end{aligned}
$$

This proof figure $\pi$ is transformed into:

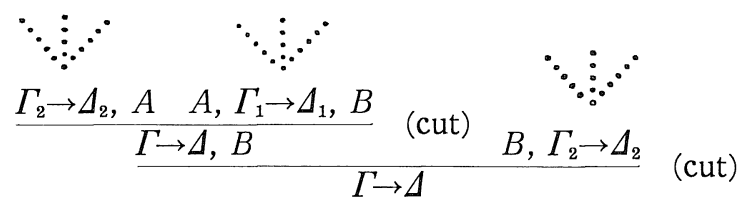

Since the grades of $A$ and $B$ are smaller than that of $A \supset B$, the induction process works well.

(2) $\rho(\pi)>2$ : There are several nontrivial cases, which we shall consider case by case in the following:

(2a) $\pi$ is of the following form:

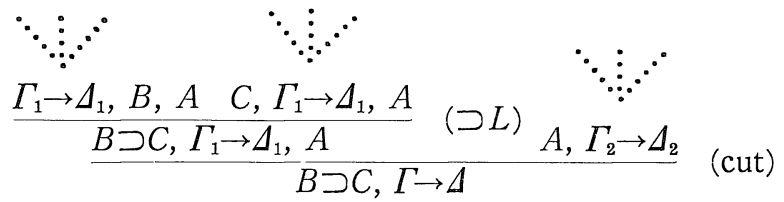

$$
\begin{aligned}
& \left(\Gamma=\Gamma_{1} \cup \Gamma_{2} \text { and } \Delta=\Delta_{1} \cup \Delta_{2}\right)
\end{aligned}
$$

1)-4) Repetition is not counted. 
Consider the following proof figure $\pi_{1}$ :

$$
\begin{aligned}
& \because \because \vdots 0^{\circ} \quad \because \because \vdots 0^{\circ} \\
& \frac{\Gamma_{1} \rightarrow \Delta_{1}, B, A \quad A, \Gamma_{2} \rightarrow \Delta_{2}}{\Gamma \rightarrow \Delta, B} \quad \text { (cut) }
\end{aligned}
$$

Since $\gamma\left(\pi_{1}\right)=\gamma(\pi), \delta\left(\pi_{1}\right) \leqq \delta(\pi)$ and $\rho\left(\pi_{1}\right)<\rho(\pi)$, we have a cut-free proof $\pi_{1}^{\prime}$ of the sequent $\Gamma \rightarrow \Delta, B$ by induction hypothesis.

Consider the following proof figure $\pi_{2}$ :

$$
\begin{gathered}
\because \because \vdots 0^{\circ} \quad \because \because 0^{\circ} \\
\frac{C, \Gamma_{1} \rightarrow \Delta_{1}, A \quad A, \Gamma_{2} \rightarrow \Delta_{2}}{C, \Gamma \rightarrow \Delta} \quad \text { (cut) }
\end{gathered}
$$

Since $\gamma\left(\pi_{2}\right)=\gamma(\pi), \delta\left(\pi_{2}\right) \leqq \delta(\pi)$ and $\rho\left(\pi_{2}\right)<\rho(\pi)$, we have a cut-free proof $\pi_{2}^{\prime}$ of the sequent $C, \Gamma \rightarrow \Delta$ by induction hypothesis. Therefore

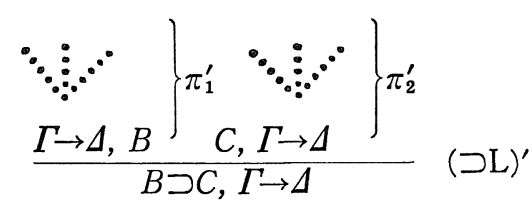

The following three cases are treated similarly to (2a).

(2b) $\rho_{l}(\pi) \geqq 2$ and the last inference of the proof of the left upper sequent of (cut) is $(\supset R)$.

(2c) $\rho_{r}(\pi) \geqq 2$ and the last inference of the proof of the right upper sequent of (cut) is $(\supset \mathrm{L})^{\prime}$.

(2d) $\rho_{r}(\pi) \geqq 2$ and the last inference of the proof of the right upper sequent of (cut) is ( $\supset R)$.

(2e) The last inference of the proofs of both upper sequents of (cut) is (GL4.3) :

We deal with the following special case, leaving the general treatment to the reader.

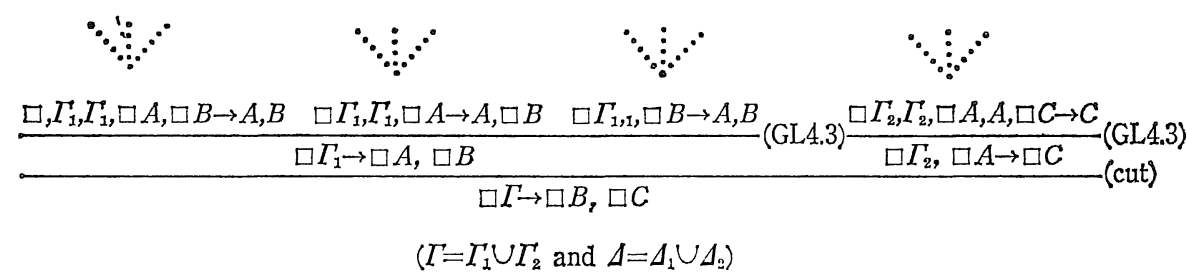


Consider the following proof figure $\pi_{1}$ :

$$
\begin{array}{cc}
\because \because \vdots 0^{\circ} & \because \because \ddots^{\circ} \\
\frac{\square \Gamma_{1}, \Gamma_{1}, \square A, \square B \rightarrow A, B \quad \square \Gamma_{2}, \Gamma_{2}, \square A, A, \square C \rightarrow C}{\square \Gamma, \Gamma, \square A, \square B, \square C \rightarrow B, C} \text { (cut) }
\end{array}
$$

Since the grade of $A$ is smaller than that of $\square A$, there is a cut-free proof $\pi_{1}^{\prime}$ of $\square \Gamma, \Gamma, \square A, \square B, \square C \rightarrow B, C$ by induction hypothesis.

Consider the following proof figure $\pi_{2}$ :

$$
\left.\frac{\because \because \vdots 0^{\circ}}{\square \Gamma_{1}, \Gamma_{1} \square B \rightarrow \square A, B \quad \square \Gamma, \Gamma, \square A, \square B, \square C \rightarrow B, C}\right\}
$$

Since $\gamma\left(\pi_{2}\right)=\gamma(\pi)$ and $\delta\left(\pi_{2}\right)<\delta(\pi)$, there is a cut-free proof $\pi_{2}^{\prime}$ of $\square \Gamma, \Gamma, \square B, \square C$ $\rightarrow B, C$ by induction hypothesis.

Consider the following proof figure $\pi_{3}$ :

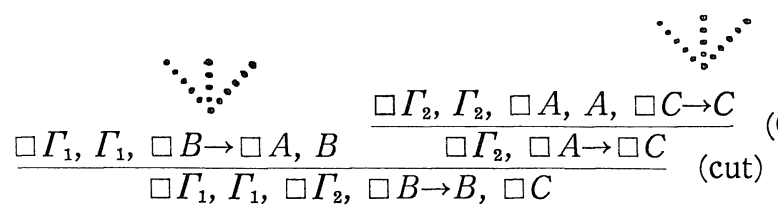

Since $\gamma\left(\pi_{3}\right)=\gamma(\pi)$ and $\delta\left(\pi_{3}\right)<\delta(\pi)$, there is a cut-free proof $\pi_{3}^{\prime}$ of $\square \Gamma, \Gamma, \square B$ $\rightarrow B, \square C$ by induction hypothesis and Lemma 3.4.

Consider the following proof figure $\pi_{4}$ :

$$
\begin{array}{cc}
\because \because \vdots 0^{\circ} & \ddots \because \vdots 0^{\circ} \\
\frac{\square \Gamma_{1}, \Gamma_{1}, \square A \rightarrow A, \square B \quad \square \Gamma_{2}, \Gamma_{2} \square A, A, \square C \rightarrow C}{\square \Gamma, \Gamma, \square A, \square C \rightarrow \square B, C} \quad \text { (cut) }
\end{array}
$$

Since $\gamma\left(\pi_{4}\right)<\gamma(\pi)$, there is a cut-free proof $\pi_{4}^{\prime}$ of $\square \Gamma, \Gamma, \square A, \square C \rightarrow \square B, C$ by induction hypothesis.

Consider the following proof figure $\pi_{5}$ :

$$
\left.\begin{array}{c}
\because \because \vdots 0^{\circ} \quad \\
\square \Gamma_{1} \rightarrow \square A, \square B \quad \square \Gamma, \Gamma, \square A, \square C \rightarrow \square B, C
\end{array}\right\} \text { (cut) }
$$

Since $\gamma\left(\pi_{5}\right)=\gamma(\pi)$ and $\delta\left(\pi_{5}\right)<\delta(\pi)$, there is a cut-free proof $\pi_{5}^{\prime}$ of $\square \Gamma, \Gamma, \square C$ $\rightarrow \square B, C$ by induction hypothesis. Therefore 


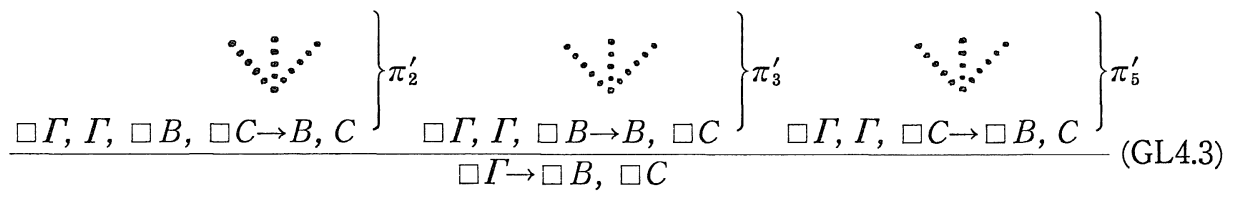

Before leaving the above proof, the reader should realize that the main reason for dealing with $\mathbf{S K} 4.3 \mathbf{G}^{\prime}$ instead of $\mathbf{S K} 4.3 \mathrm{G}$ directly is to make the secondary induction on $\delta(\pi)$ work well. It seems that the secondary induction of Theorem 3.4 (cut-elimination theorem) of Leivant [4] indeed works well for cases like (a special case of) (2e) but fails to preserve the usual treatment of classical cases like (2c).

\section{References}

[1] Boolos, G., The unprovability of consistency, Cambridge University Press, 1979.

[2] Gabbay, D.M., Investigations in modal and tense logics with applications to problems in philosophy and linguistics, D. Reidel, 1976.

[3] Goldblatt, R., Arithmetical necessity, provability and intuitionistic logic, Theoria, 44 (1978), 38-46.

[4] Leivant, D., On the proof theory of the modal logic for arithmetical provability, J. Symbolic Logic, 46 (1981), 531-538.

[5] Sato, M., A cut-free Gentzen-type system for the modal logic S5, J. Symbolic Logic, 45 (1980), 67-84.

[6] Takeuti, G., Proof theory, North-Holland, 1975.

[7] Zeman, J. J., Modal logic, Oxford University Press, 1973. 